\title{
Cómo como - cómo soy - cómo me veo. Un estudio con niños y niñas que juegan al fútbol
}

\author{
Adriano FERNÁNDEZ VALLINES \\ Carmen GONZÁLEZ GONZÁLES DE MESA
}

Datos de contacto:

Adriano Fernández Vallines adri14vallines@gmail.com Universidad de Oviedo, España.

Carmen González González de Mesa gmcarmen@uniovi.es Universidad de Oviedo, España.

\section{RESUMEN}

Un alto porcentaje de los niños en etapa primaria de la educación española realizan como actividad extraescolar la práctica de uno o varios deportes para aumentar las horas semanales de actividad física. Esto unido a los hábitos alimentarios nos dan una imagen clara de cómo está cambiando la sociedad española en lo referente a salud infantil. El principal objetivo de este estudio es relacionar el Autoconcepto, los Hábitos Alimentarios, la Imagen Corporal y las horas semanales que dedican a realizar Actividad Física en niños y niñas que ya realizan fútbol como actividad extraescolar. Los participantes de este estudio han sido 124 escolares entre 8 a 13 años del Principado de Asturias. Se aplicaron los instrumentos Escala de autoconcepto físico para jóvenes (C-PSQ), una parte de Multidimensional Body Self Relations Questionnaire (MBSRQ), Test de Evaluación de Preferencias, Intenciones de Conducta y Consumos Alimentarios (TEPICA) y Siluetas de Collins. Se han realizado estadísticos Descriptivos, Pruebas $t$ de Student para muestras relacionadas e independientes, Análisis de Varianza y Correlación de Pearson. Los resultados mostraron que quién tiene conocimientos adecuados sobre hábitos alimentarios se correlaciona positivamente con el nivel de autoconcepto, que las horas de práctica deportiva se correlacionan positivamente con la competencia percibida y la autoconfianza, y que, aunque manifiestan estar satisfechos con las diferentes partes del cuerpo, les gustaría estar más delgados que como se ven a sí mismos.

PALABRAS CLAVE: Deporte extraescolar; Autoconcepto; Imagen corporal; Hábitos alimentarios; Índice de masa corporal. 


\title{
How I eat - how I am - how I look. A study with boys and girls who play football
}

\begin{abstract}
A high percentage of children in the primary stage of Spanish education perform as an extracurricular activity the practice of one or more sports to increase the weekly hours of physical activity. This together with eating habits give us a clear picture of how Spanish society is changing in relation to children's health. The main goal of this study is to relate the self-concept, the nutritional habits, the body image and the numbers of hours that children who already play football spend doing physical activity per week. The number of children that took part in this study was a sample of 124 schoolchildren in between 8 and 13 years old, of the Principality of Asturias. The surveys done for this study were Youth Physical Self-Concept Scale (C-PSQ), Multidimensional Body Self Relations Questionnaire (MBSRQ), Test Evaluation Preferences, Intentions of Conduct and Food Consumption (TEPICA) and Collins Silhouettes. Descriptives statistics were done, T-student tests for related and independent samples, variance analysis and Pearson correlation. The results showed that children who have good knowledge of nutricional habits correlates positively with the self-concept level. The results also show that the number of hours doing sports correlates positively with the perceived competency and self- concept. Also those children that aparently are satisfy with their bodies would like to be thiner.
\end{abstract}

KEYWORDS: Out-of-school sport; Selfconcept; Body image; Eating habits; Body mass index.

\section{Introducción}

Existen muchas investigaciones sobre la actividad fisica (AF) en la etapa primaria y sobre su repercusión en el alumno. Este estudio mostrará su relación con los conocimientos y hábitos alimentarios, el autoconcepto físico, el índice de masa corporal (IMC) y la imagen corporal de los escolares participantes. En este trabajo ya está implícita la actividad física porque los participantes practican regularmente fútbol extraescolar.

Dándole un enfoque más clínico a lo que supone la práctica de AF y sus beneficios y riesgos, cabe citar el estudio de Hanifi, Lamber y Haskell (2010), en el que enumeran los factores de riesgo más importantes de mortalidad en el mundo, ocupando el cuarto lugar la inactividad física (6\%), seguido del sobrepeso y la obesidad (5\%). Cenarruzabeitia, Hernández y Martínez (2003), apoyados por una rigurosa investigación epidemiológica, afirman que la práctica de AF protege de cardiopatía isquémica, hipertensión arterial y accidentes cerebrovasculares.

Para que la AF produzca beneficios en la salud, mejorando las funciones cardiorrespiratorias y musculares, Hanifi et al. (2010) recomiendan 60 
minutos diarios de actividad fisica de intensidad moderada/vigorosa, practicar diariamente $\mathrm{AF}$ aeróbica $\mathrm{y}$ tres veces por semana actividades vigorosas.

Conociendo la importancia y virtudes que tiene la AF, otro problema que acecha a la población infantil son las nuevas formas de ocio, que en la actualidad son mucho más sedentarias que las del pasado, con el riesgo que ello produce en estas edades (Busto, Amigo, Herrero y Fernández, 2006). La desinformación de las familias y la poca importancia que se da desde los centros escolares al descanso, hábitos saludables y $\mathrm{AF}$, hace que últimos estudios adviertan de los riesgos que ocasiona, como por ejemplo la investigación de Soler y Castañeda (2017) que explican las complicaciones de salud infantil asociados al sedentarismo, señalando como principal problema la obesidad, y confirmando que la práctica de AF beneficia la salud y contribuye a contrarrestar el sedentarismo.

El futbol, deporte practicado por los participantes en el estudio, es el deporte por excelencia en España y el más practicado en todas las franjas de edad. El Consejo Superior de Deportes, en el Anuario de Estadísticas Deportivas 2017 realizado por la Subdirección General de Estadística y Estudios de la Secretaría General Técnica del Ministerio de Educación, Cultura y Deporte, refleja de forma numérica la fuerza que tiene el futbol y el número de personas que lo practican, indicando que en 2016 el número de licencias federativas en hombres es 2.814 .387 (78.5\%), y el de mujeres 771.746 (21.5\%), alcanzando un $26.3 \%$ del total de licencias deportivas en España. Practicado en edades tempranas como actividad extraescolar, debería tener un gran poder formativo y social, un lugar donde cooperen y compitan, respeten normas y se sientan parte de un equipo. Si se enfocara adecuadamente llevaria a conductas positivas, como pueden ser el respeto, compañerismo, disciplina, etc. Es fundamental entrelazar el ámbito escolar (formativo formal) y extraescolar (formativo deportivo) para una educación holística (Merino-Orozco, Arraiz-Pérez, Sabirón-Sierra, Usán-Supervía y Jarie, 2017).

Existen más estudios con experiencias positivas que aportan información relevante sobre las virtudes y oportunidades del fútbol en la etapa escolar, por ejemplo los que indican que practicar futbol extraescolar aumenta la motivación del alumno (Hortigüela, Fernández-Río y Pérez, 2016), o que las actitudes y valores positivos desarrollados en el futbol repercutan en el aula, por ejemplo trabajo cooperativo, comunicación, control de contingencias... (Garcia, López y Eslava-Suanes, 2018).

Según Cuervo, Cachón, Zagalaz y González (2018), con la práctica de actividad fisica se aumenta el interés hacia una alimentación saludable, afirmación relevante al considerar que los conocimientos erróneos sobre alimentación están muy extendidos en la sociedad (Murillo-Godínez y PérezEscamilla, 2017).

Para una visión general sobre lo relacionado con hábitos saludables, alimentación y actividad física, se utilizara como fuente fidedigna las 
recomendaciones la OMS (Organización Mundial de la Salud), organismo de la Organización de las Naciones Unidas (ONU) especializado en gestionar politicas de prevención, promoción e intervención en salud a nivel mundial. Los estudios presentados arrojan datos preocupantes sobre la realidad presente y futura en relación al peso y los hábitos saludables de las nuevas generaciones. En recientes escritos manifiestan que "La obesidad en la población infantil y adolescente se ha multiplicado por diez en los últimos cuatro años", y según un reciente estudio del Imperial College de Londres y la OMS, se prevé que en el año 2022 "habrá más niños y adolescentes con obesidad que con insuficiencia ponderal". La OMS $(2016,2017)$ sostiene que el aumento del IMC alto es un factor de riesgo de enfermedades no transmisibles, como las cardiovasculares, diabetes, las del aparato locomotor, determinados cánceres...

En este trabajo se estudia también el autoconcepto y su importancia afectiva y motivacional, constructo de máxima importancia en el desarrollo de la personalidad en edades preadolescentes. Autores como González-Pienda, Núñez, González-Pumariega y García (1997) destacan el papel que ocupan en la regulación de estrategias cognitivo-emocionales implicadas en el aprendizaje y el rendimiento académico. Goñi e Infante (2015) señalan que la práctica de actividad fisica hace mejorar el autoconcepto y los niveles de satisfacción con la vida.

También en este estudio se tiene en cuenta la Imagen Corporal, constructo entendido por Schilder (2013) como la autopercepción de la IC. La primera relación que las personas tienen de la IC (Jiménez-Flores, JiménezCruz y Bacardi-Gascón, 2017) comienza a los 5 años, y manifiestan que los niños sienten insatisfacción, preocupación y percepción inexacta de ella. En lo referente al apartado psicológico cabe destacar que la IC no está necesariamente correlacionada con la apariencia fisica, siendo un elemento clave la valoración personal, y si ésta valoración difiere con la realidad puede presentar alteraciones de la personalidad (Sepúlveda, Gandarillas y Carrobes, 2004). La práctica de AF permite el crecimiento corporal y expresivo, siendo una herramienta potencial para mejorar la IC (Vaquero, Macías y Macazaga, 2014).

Así las cosas, en el apartado siguiente se mostrarán la metodología y los resultados de este trabajo.

\section{Método}

\section{Objetivos}

El objetivo de este trabajo ha sido estudiar el autoconcepto físico, los conocimientos y hábitos alimentarios, el índice de masa corporal y la imagen corporal de los preadolescentes que juegan al fútbol de manera extraescolar y las posibles inferencias y relaciones entre las diferentes variables objeto de estudio. 


\section{Participantes}

Los participantes en el estudio han sido 124 preadolescentes asturianos que entrenan fútbol de manera extraescolar (11 mujeres y 113 varones). El rango de edad oscila entre 8 a 13 años, cuya media de edad es de 10.40 años, de los cuales 64 están entre 8 y 10 años y 60 mayores de 10 años. Las horas semanales de práctica deportiva varian entre 1 y 15 horas o más, y se han concentrado en tres grupos, los que hacen poco deporte (de 1 a 3 horas semanales), los que practican deporte de manera moderad (de 4 a 6 horas), y los que hacen mucho deporte (más de 6 horas semanales).

\section{Instrumentos}

Se ha aplicado un cuestionario con escalas previamente validadas en investigaciones anteriores realizadas por otros autores, y precedido por variables personales y familiares.

La adaptación del Test de Evaluación de Preferencias, Intenciones de Conducta y Consumos Alimentarios (TEPICA), (Benarroch y Pérez, 2011; Benarroch, Pérez y Perales, 2011; Pérez-Vadillo, 2013), revalidado por Cuervo (2016) en su tesis doctoral. Inicialmente, esta escala era mucho más amplia, en la adaptación de Cuervo (2016) se descartaron variables, bien porque no tenían relación con el estudio y los objetivos del trabajo, como es ahora el caso, o por los malos resultados en el análisis factorial. Las respuestas son escala Likert de 1 a 5 puntos según el grado de acuerdo o desacuerdo con cada uno de los ítems.

El Test de Siluetas de Collins (1991) que fue creado a partir de las siluetas de Stunkard, Sorensen y Schlusinger (1983). Se muestran 7 siluetas solicitando que evalúen la percepción de su imagen corporal. Las siluetas avanzan de izquierda a derecha de más delgado/a hasta más ancho/a. Las dos preguntas a contestar son ¿Cómo me veo? y ¿Cómo me gustaría ser? (Muñoz, 2015) y deben señalar la figura con la que se identifican.

La Escala de autoconcepto físico para jóvenes (C-PSQ) de Fox y Corbin (1989), adaptada por Moreno, Cervelló, Vera y Ruiz (2007). Los ítems evalúan la competencia percibida, atractivo fisico, fuerza física y la autoconfianza. Se valoran mediante una escala Likert del 1 al 5, siendo 1 la puntuación correspondiente a "Totalmente en desacuerdo" y 5 "Totalmente de acuerdo".

Por último, una parte del Multidimensional Body Self Relations Questionnaire, (MBSRQ), que en su versión original (Brown, Cash y Mikulka, 1990; Cash, 1990, 2000) constaba de 69 ítems, configurados en diez factores. Ha sido validado en población española por Botella, Ribas, y Benito (2009) para adultos, reduciendo la escala a 45 ítems. Finalmente se utilizó la adaptación de Coballes (2015) que en su tesis doctoral, ya que lo adaptó y validó para escolares preadolescentes, lo redujo a 23 variables y 3 factores. 
En este trabajo solo se utilizó el primer factor que mide el grado de satisfacción con diferentes partes del cuerpo.

También se han medido y pesado a todos los participantes para calcular el IMC.

\section{Procedimiento}

Para seleccionar a los participantes, se ha enviado una solicitud avalada por Facultad de Formación del Profesorado y Educación de la Universidad de Oviedo, a varios clubs en los que se practica el fútbol de manera extraescolar.

Una vez obtenida la aprobación y permisos, se ha contactado con las familias de los jugadores para explicar la finalidad del estudio y solicitar autorización.

En horario de entrenamiento y en los días concertados, los autores de este trabajo, han aplicado los cuestionarios, aclarando las dudas surgidas y asegurando la veracidad, confidencialidad y libre elección de las respuestas. Los cuestionarios se distribuyeron en tandas de cuatro jugadores para tener control y facilitarles la realización de los cuestionarios. La media de realización de la prueba, entre cuestionarios y mediciones osciló entre 20 o 30 minutos, dependiendo de la franja de edad y conocimientos. Las mediciones de peso y altura fueron realizadas in situ seguidamente a la aplicación de los cuestionarios.

\section{Análisis Estadístico}

Una vez vaciado los datos en el paquete estadístico SPSS.24, se ha recodificado y creado algunas variables antes de proceder al análisis estadístico, reconvirtiendo los items siguientes:

- Las preguntas formuladas en negativa a preguntas en positivo.

- Las edades en dos grupos (pequeños de 8-10 y mayores de 11-13).

- Los valores del test de imágenes de 7 a 5 valores (fueron pocas personas las que contestaron los valores extremos).

- La valoración de las diferentes partes del cuerpo a valoración dicotómica, satisfechos-insatisfechos.

- Los valores del IMC por cuartiles.

- La cantidad de práctica deportiva semanal agrupado las horas en tres valores (poco deporte: 1- 3 horas, deporte moderado: 4- 6 horas y mucho deporte: más de 6 horas).

Se han calculado los factores de las dos escalas y se ha procedido al análisis descriptivo (tendencia central y dispersión), correlaciones bivariadas (Correlación de Pearson), comparación de medias (muestras relacionadas) y análisis inferencial ( $t$ de Student y ANOVA). 


\section{Resultados}

Se comenzará explicando los resultados descriptivos de los datos personales y familiares. Tablas 1 y 2 .

En lo referente al número de ingestas diarias el mayor número de participantes concentran sus respuestas en 5 ingestas (56.5\%) y 4 (30.6\%) ingestas diarias. Al ítem quien cocina en casa los datos nos dicen que la mitad de los encuestados cocina solo la madre, el $34.7 \%$ los dos progenitores y el $8.1 \%$ solo el padre.

En los items que hacen referencia a la familia, el 80,6\% viven con sus padres, aunque eso no implique el mismo domicilio familiar. El número de hermanos se concentra en dos con un $56.5 \%$, y un $28.2 \%$ son hijos únicos, muy alejados de las demás opciones. En relación con la ocupación laboral el $61.3 \%$ de los encuestados solo trabaja el padre fuera de casa y el $21.8 \%$ ambos progenitores. Hay cinco familias en las que no trabaja nadie.

En relación a la pregunta si practican otro deporte reglado aparte del fútbol, el $73.4 \%$ de los encuestados solo practican fútbol federado y el $26.6 \%$ practican también otro deporte de forma regular y reglada.

A continuación, se ha procedido a emparejar los datos obtenidos de los valores entre "cómo se ven" (D1) y "cómo les gustaría ser" (D2). Se ha calculado el estadístico $t$ para muestras relacionadas, obteniendo como resultado que la media otorgada es más alta a favor de "cómo se ven", con una $\mathrm{DM}=.379$, siendo estos resultados estadísticamente significativos $(t=5.013, p<.001)$.

En el cuestionario de Autoconcepto Físico, se ha transformado los items formulados en negativo para que todos vayan en la misma línea. Estos se han señalado en cursiva en la tabla 1.

Se muestran a continuación los resultados del análisis descriptivo (tendencia central y dispersión) de los Ítems de los dos cuestionarios, observando que la distribución es normal, no siendo necesario prescindir de ningún ítem, los valores de asimetría y curtosis no difiere de \pm 4.0 , por tanto, los estadísticos paramétricos son suficientemente robustos a la desviación de la normalidad (Schmider, Ziegler, Danay, Beyer y Bühner, 2010). 
Tabla 1

\begin{tabular}{|c|c|c|c|c|}
\hline & $\mathbf{M}$ & DT & Asim. & Curt. \\
\hline 1. Soy muy bueno/a en casi todos los deportes (COM.PERC) & 3.58 & .847 & -.216 & -.112 \\
\hline Siempre mantengo una excelente forma fisica (COM.PERC) & 3.73 & 1.015 & -.326 & -.588 \\
\hline 3. Comparado con la mayoría, mi cuerpo no es tan bonito (ATRC.FIS) & 2.50 & 1.144 & .281 & -.699 \\
\hline $\begin{array}{l}\text { 4. Comparado con la mayoría de la gente de mi mismo sexo, creo que } \\
\text { me falta fuerza fisica (FUER.FIS) }\end{array}$ & 2.45 & 1.303 & .231 & -1.279 \\
\hline $\begin{array}{l}\text { 5. Me siento muy orgulloso/a de lo que soy y de lo que puedo hacer } \\
\text { fisicamente (AUTOCONF) }\end{array}$ & 4.38 & .916 & -1.603 & 2.354 \\
\hline $\begin{array}{l}\text { 6. Siempre me organizo para poder hacer ejercicio fisico intenso de } \\
\text { forma regular y continuada (COM.PERC) }\end{array}$ & 3.85 & .996 & -.438 & -.444 \\
\hline 7. Tengo dificultad para mantener un cuerpo bonito (ATRC.FIS) & 3.27 & 1.001 & -.623 & 1.024 \\
\hline $\begin{array}{l}\text { 8. Mis músculos son tan fuertes como los de la mayoría de las } \\
\text { personas de mí mismo sexo (FUER.FIS) }\end{array}$ & 3.52 & 1.199 & -.561 & -.502 \\
\hline 9. Siempre estoy satisfecho/a de cómo soy fisicamente (ATRC.FIS) & 4.20 & .928 & -.973 & .294 \\
\hline $\begin{array}{l}\text { 10. No me siento seguro/a cuando se trata de participar en actividades } \\
\text { deportivas (AUTOCONF) }\end{array}$ & 4.57 & .677 & -1.468 & 1.439 \\
\hline $\begin{array}{l}\text { 11. Siempre mantengo un alto nivel de resistencia y forma fisica } \\
\text { (COM.PERC) }\end{array}$ & 3.99 & .870 & -.739 & .795 \\
\hline $\begin{array}{l}\text { 12. Me siento avergonzado/a de mi cuerpo cuando se trata de llevar } \\
\text { poca ropa (ATRC.FIS) }\end{array}$ & 2.06 & 1.271 & .932 & -.288 \\
\hline $\begin{array}{l}\text { 13. Cuando se trata de situaciones que requieren fuerza, soy el } \\
\text { primero/a en ofrecerme (FUER.FIS) }\end{array}$ & 3.52 & 1.193 & -.409 & -.680 \\
\hline $\begin{array}{l}\text { 14. Cuando se trata del aspecto físico, no siento mucha confianza en mí } \\
\text { mismo (AUTOCONF) }\end{array}$ & 2.33 & 1.330 & .488 & -1.077 \\
\hline $\begin{array}{l}\text { 15. Considero que siempre soy de los/as mejores cuando se trata de } \\
\text { participar en actividades deportivas (COM.PERC) }\end{array}$ & 3.69 & .973 & -.696 & .153 \\
\hline $\begin{array}{l}\text { 16. Suelo encontrarme un poco incómodo/a en lugares donde se practica } \\
\text { ejercicio físico y deporte (COM.PERC) }\end{array}$ & 1.77 & 1.250 & 1.546 & 1.153 \\
\hline $\begin{array}{l}\text { 17. Pienso que a menudo se me admira porque mi fisico o mi tipo de } \\
\text { figura se considera bonita (ATRC.FIS) }\end{array}$ & 2.71 & 1.065 & -.010 & -.416 \\
\hline $\begin{array}{l}\text { 18. Tengo poca confianza cuando se trata de mi fuerza física } \\
\text { (AUTOCONF) }\end{array}$ & 2.00 & 1.243 & .956 & -.291 \\
\hline $\begin{array}{l}\text { 19. Siempre tengo un sentimiento verdaderamente positivo de mi } \\
\text { aspecto fisico (AUTOCONF) }\end{array}$ & 4.15 & .911 & -.769 & -.080 \\
\hline $\begin{array}{l}\text { 20. Suelo estar entre los/as más rápidos/as cuando se trata de } \\
\text { aprender nuevas habilidades deportivas (AUTOCONF) }\end{array}$ & 3.94 & .990 & -.600 & -.457 \\
\hline $\begin{array}{l}\text { 21. Me siento muy confiado/a para practicar de forma continuada y } \\
\text { para mantener mi forma fisica (COM.PERC) }\end{array}$ & 4.07 & .876 & -.585 & -.146 \\
\hline $\begin{array}{l}\text { 22. Creo que, comparado/a con la mayoría, mi cuerpo no parece estar } \\
\text { en la mejor forma (COM.PERC) }\end{array}$ & 2,45 & 1,205 &, 427 &,- 710 \\
\hline $\begin{array}{l}\text { 23. Creo que, comparado con la mayoría, soy muy fuerte y tengo } \\
\text { músculos bien desarrollados (FUER.FIS) }\end{array}$ & 3,28 & 1,116 &,- 294 &,- 558 \\
\hline 24. Desearia tener más respeto hacia mi propio fisico (ATRC.FIS) & 2,73 & 1,362 & 240 & $-1,017$ \\
\hline $\begin{array}{l}\text { 25. Cuando surge la oportunidad, siempre soy de los/as primeros/as } \\
\text { para participar en deportes (COM.PERC) }\end{array}$ & 4,31 & ,912 & $-1,303$ & 1,190 \\
\hline 26. No me siento seguro/a sobre el aspecto de mi cuerpo (ATRC.FIS) & 1,93 & 1,156 & 1,075 & 321 \\
\hline $\begin{array}{l}\text { 27. Creo que no soy tan bueno/a como la mayoría cuando se trata de } \\
\text { situaciones que requieren fuerza (FUER.FIS) }\end{array}$ & 2,47 & 1,297 &, 504 &,- 806 \\
\hline $\begin{array}{l}\text { 28. Me siento muy satisfecho/a tal y como soy fisicamente } \\
\text { (AUTOCONF) }\end{array}$ & 4,22 & 1,017 & $-1,253$ & 1,012 \\
\hline
\end{tabular}


Tabla 2

\section{Hábitos Alimentarios}

1. Creo que el agua engorda si la tomamos entre las comidas (M)

M DT $\quad$ ASIM. CURT.

2. El alcohol es un nutriente energético que debe ser consumido con moderación en una dieta equilibrada (M)

3. Las hamburguesas, pizzas y similares alimentan más que las comidas que se hacen en casa $(\mathrm{M})$

4. El consumo de pescado azul protege al corazón de las enfermedades cardiovasculares (CA)

5. Las frutas tomadas después de las comidas producen fermentaciones que son perjudiciales para la salud y engordan (M)

6. Creo que el vino antes de las comidas abre el apetito y da fuerzas (M)

7. Los niños sólo deben tomar pescado blanco (M)

1.73

1.127

$\begin{array}{llll}1.90 & 1.324 & 1.200 \quad .114\end{array}$

8. Se debe comer pescado tres o cuatro veces a la semana (CA)

9. Una alimentación adecuada durante la adolescencia previene la aparición de algunas patologías en la edad adulta (CA)

10. Hay que comer frutas y verduras todos los días (CA)

$\begin{array}{llll}1.79 & 1.128 & 1.287 & .762\end{array}$

$3.92 \quad .959 \quad-.174 \quad-1.063$

$1.97 \quad 1.140 \quad 834 \quad-173$

1. Es lo mismo tomar zumo de naranja natural, refrescos de naranja o caramelos enriquecidos con vitamina (CA)

\begin{tabular}{rrrr}
1.97 & 1.140 & .834 & -.173 \\
\hline & &
\end{tabular}

12. Es mejor comer lo que comen los demás y no destacar (M)

13. La delgadez extrema está de moda, pero no equivale a tener buena salud (CA)

$2.09 \quad 1.162 \quad .678 \quad-.350$

\begin{tabular}{|c|c|c|c|}
\hline 1.80 & 1.036 & .951 & -288 \\
\hline
\end{tabular}

$3.63-1.122--.487 \quad-.416$

$3.39 \quad 1.383 \quad-.408 \quad-.917$

$4.68 \quad .669-1.999 \quad 2.996$

$\begin{array}{llll}2.40 & 1.325 & .458 & -.928\end{array}$

14. Como pocas frutas porque eso no se lleva entre mis colegas (M)

\begin{tabular}{|c|c|c|c|}
\hline 2.34 & 1.195 & .448 & -.659 \\
\hline
\end{tabular}

15. En los anuncios de televisión siempre salen chicos/as muy delgados porque es así como debemos estar todos (M)

$\begin{array}{llll}3.48 & 1.445 & -.534 & -.994\end{array}$

$\begin{array}{llll}1.69 & 1.039 & 1.460 & 1.340\end{array}$

16. Siento que los alimentos controlan mi vida (M)

17. En casa carecemos de recursos económicos para comprar pescado, frutas y verduras (M)

18. En mi casa siempre comemos comida precocinada o un bocata (M)

$\begin{array}{llll}2.15 & 1.288 \quad .869 & -.270\end{array}$

$\begin{array}{cccc}2.68 & 1.322 & .187 & -1.020 \\ 2.04 & 1.388 & .966 & -.494\end{array}$

19. En mi casa casi nunca comemos juntos (M)

20. Evito especialmente comer alimentos con muchos hidratos de carbono (por ejemplo, pan, arroz, patatas, etc.) (M)

21. Suelo comer siempre los mismos alimentos $(\mathrm{M})$

22. Me siento incómodo/a después de comer dulces (M)

23. Tengo fácil acceso a fruta y verdura variada (CA)

$1.63 \quad 1.093 \quad 1.809 \quad 2.360$

24. Creo que la ingesta diaria de alimentos ricos en grasas y azúcares perjudica la salud (CA)

25. Creo que tengo suficientes conocimientos acerca de los efectos beneficiosos de llevar una dieta equilibrada (CA)

26. Sé distinguir los alimentos ricos en grasas, colesterol, azúcares añadidos y sal de los que no lo son (CA)

\begin{tabular}{cccc}
\hline 1.66 & 1.081 & 1.614 & 1.698 \\
\hline 2.47 & 1.246 & .357 & -.829 \\
\hline 2.15 & 1.234 & .800 & -.424 \\
\hline 2.56 & 1.410 & .387 & -1.148 \\
\hline 4.06 & 1.153 & -1.131 & .569 \\
\hline 3.89 & 1.295 & -.927 & -.134 \\
3.77 & 1.073 & -.619 & -.085 \\
\hline 3.52 & 1.310 & -.517 & -.663 \\
\hline 3.11 & 1.283 & -.073 & -.789 \\
\hline 1.96 & 1.290 & 1.115 & -.052 \\
2.02 & 1.340 & 1.067 & -.095 \\
\hline 4.36 & .940 & -1.565 & 2.320 \\
\hline
\end{tabular}

27. El vegetarianismo estricto es una manera sana de perder peso (M)

28. Raras veces veo a mis padres comer futas o verduras (M)

29. A mi padre le encanta ver la tele comiendo pipas y bebiendo una coca cola $(\mathrm{M})$

30. Para tener una dieta equilibrada se aconseja seguir las indicaciones de la pirámide de los alimentos (CA)

Se ha procedido a calcular los diferentes factores que configuran los cuestionarios (se muestra entre paréntesis las siglas con las que se nombrarán a partir de ahora). Ver tabla 3. Factores del Autoconcepto Físico (C-PSQ): 
- Competencia percibida (COM.PERC)

- Atractivo fisico (ATRC.FIS)

- Fuerza física (FUER.FIS)

- Autoconfianza (AUTOCONF)

Factores de Hábitos Alimentarios (TEPICA):

- Conocimientos y creencias erróneas (C.ERRO)

- Conocimientos y creencias adecuadas (C.ADEC)

El factor que más alto han puntuado es AUTOCONF y el menos C.ERRO. Los resultados de las pruebas Asimetría y Curtosis son adecuadas, solo supera el valor 1.00 la Curtosis del factor C.ERRO.

Tabla 3

Tendencia central y dispersión de los factores

\begin{tabular}{lcccc}
\hline FACTORES & M & DT & Asim. & Curt. \\
\hline COM.PERC & 2.62 & .454 & .708 & .36 \\
ATRC.FIS & 3.27 & .504 & .011 & .559 \\
FUER.FIS & 3.19 & .498 & .193 & -.015 \\
AUTOCONF & 3.71 & .536 & -.598 & .34 \\
C.ERRO & 2.52 & .402 & .736 & 1.342 \\
C.ADEC & 3.13 & .421 & -.027 & .602 \\
\hline
\end{tabular}

Seguidamente se han calculado las correlaciones bivariadas aplicando el estadístico correlación de Pearson entre factores, las horas semanales de práctica deportiva y el IMC. Ver tabla 4.

Se obtienen varias correlaciones estadísticamente significativas al $99 \%$, con valores superiores al .250, la competencia percibida con el atractivo fisico (.513), con fuerza física (.318), con la autoconfianza (.719) y con conocimientos adecuados (.413), el atractivo físico correlaciona con la autoconfianza (.506), la fuerza física con la autoconfianza (.376) y con los conocimientos adecuados (.256) y la autoconfianza con los conocimientos adecuados (.434).

Cabe destacar que los conocimientos erróneos correlacionan de manera estadísticamente significativa, pero en sentido negativo, con la competencia percibida, el atractivo físico y la autoconfianza, lo cual indica que los que puntúan más alto en conocimientos y creencias erróneas, puntúan menos en estos tres factores y viceversa.

También se puede observar que las horas de entrenamiento deportivo correlacionan estadísticamente significativo en un $95 \%$ con la competencia percibida y la autoconfianza (.192 y .212 respectivamente). 
Cómo como - cómo soy - cómo me veo. Un estudio con niños y niñas que juegan al fútbol

Tabla 4

Correlaciones bivariadas de los factores estudiados, IMC y horas semanales de práctica deportiva

\begin{tabular}{|c|c|c|c|c|c|c|c|c|}
\hline & & 2 & 3 & 4 & 5 & 6 & 7 & 8 \\
\hline 1. & IMC & -.017 & .094 & -.104 & -.047 & -.032 & .127 & -.084 \\
\hline 2. & COM.PER & 1 & $.513^{* *}$ & $.318^{* *}$ & $.719^{* *}$ & $-.339^{* *}$ & $.413^{* *}$ & $.192^{*}$ \\
\hline 3. & ATRC.FIS & & 1 & .159 & $.506^{* *}$ & $-.368^{* *}$ & .136 & .007 \\
\hline & FUER.FIS & & & 1 & $.376^{* *}$ & .100 & $.256^{* *}$ & .142 \\
\hline 5. & AUTOCONF & & & & 1 & $-.388^{* *}$ & $.434^{* *}$ & $.212^{*}$ \\
\hline & CC.ERRO & & & & & 1 & -.085 & -.152 \\
\hline & CC.ADEC & & & & & & 1 & .002 \\
\hline
\end{tabular}

**. La correlación es significativa al nivel 0,01 (bilateral) $=99 \%$

*. La correlación es significante al nivel 0,05 (bilateral) $=95 \%$

En cuanto a "cómo se ven" y "cómo les gustaría verse" se ha reconvertido los valores en cinco escalas en vez de siete. Se presentan a continuación, en las figuras 1 y 2 , los resultados de las medias de los cinco factores. Se observa en "como les gustaria ser" que nadie quiere ser muy grueso.

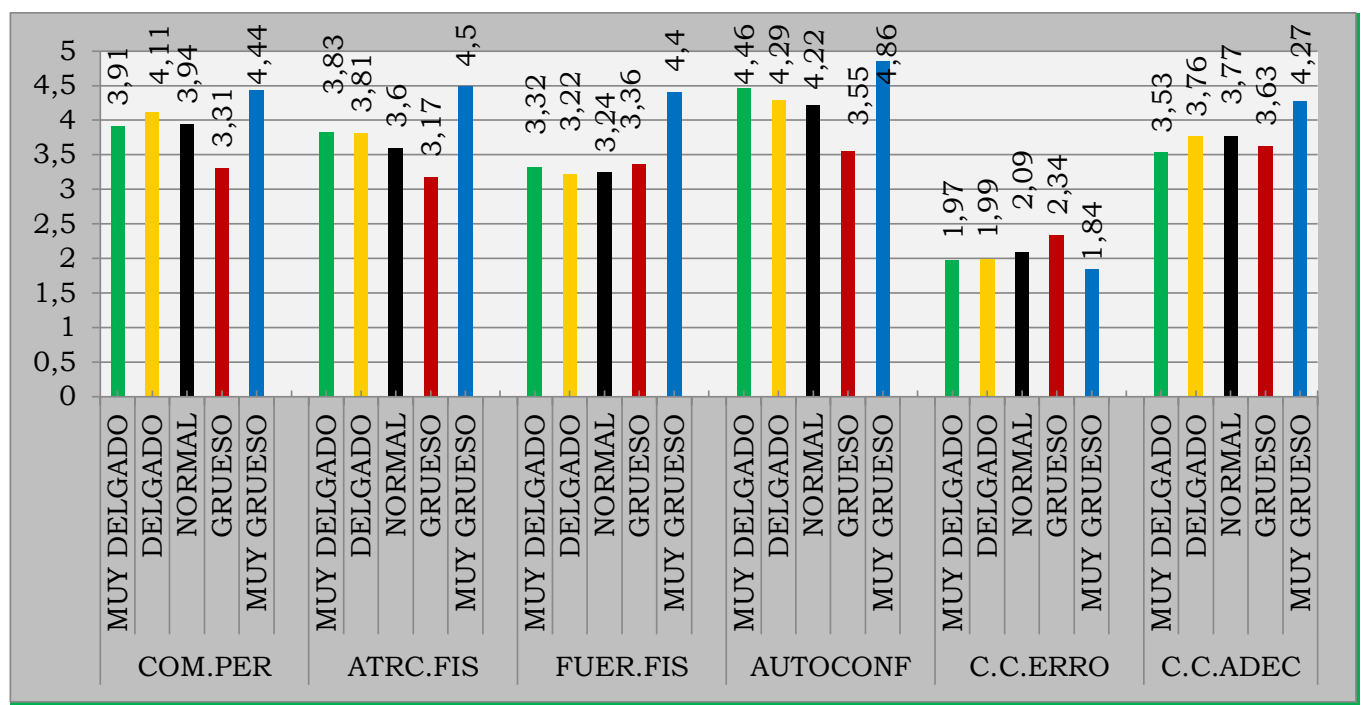

Figura 1. Comparación de las medias otorgadas a los factores con relación a "Cómo se ven" 


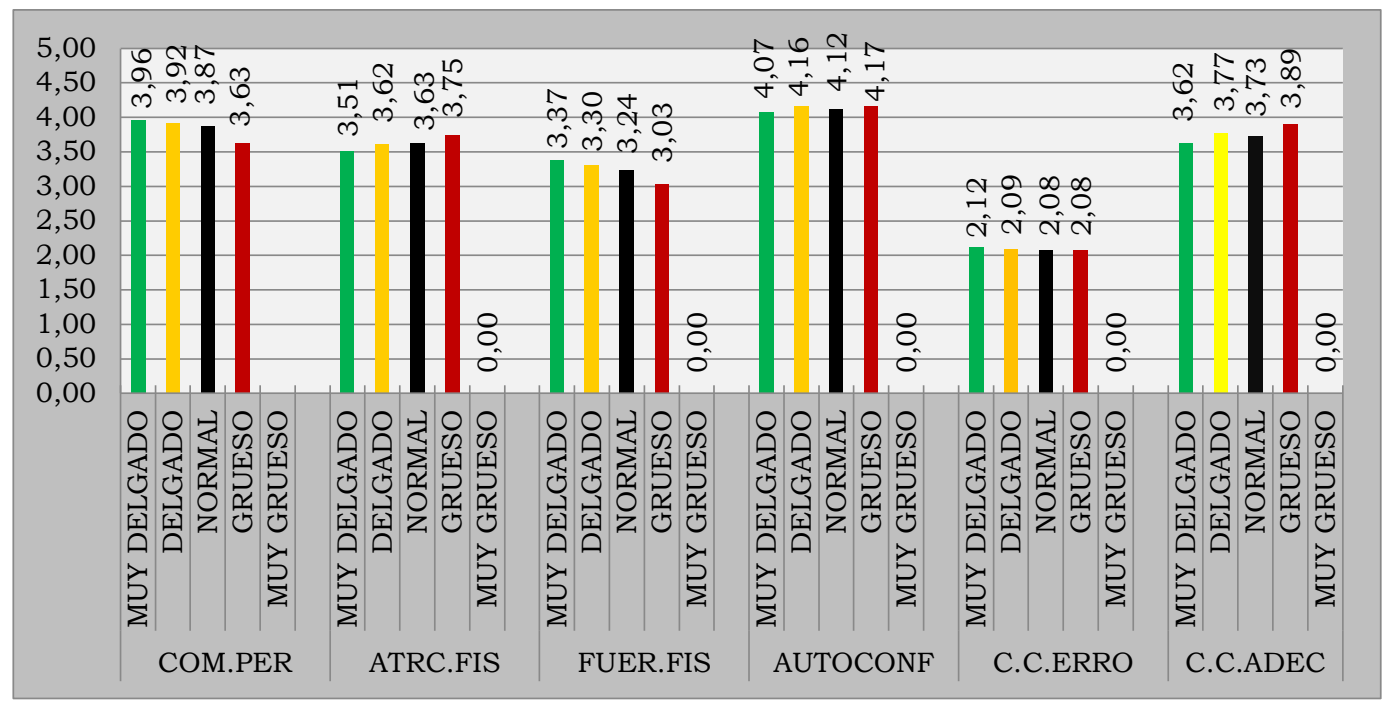

Figura 2. Comparación de las medias otorgadas a los factores con relación a "Cómo les gustaría ser"

Se realizó un análisis de varianza (ANOVA) para saber si el "cómo se ven" y "cómo les guastaría verse" infiere en los factores estudiados. Ver tabla 5.

En "cómo se ven" hay 3 factores que presentan valores estadísticamente significativo, competencias percibidas $(\mathrm{F}=10.192, p<.001)$, atractivo físico $(\mathrm{F}=5.582, p<.001)$ y autoconfianza $(\mathrm{F}=8.800, p<.001)$, mientras que en la variable "cómo me gustaría ser" los datos no resultan estadísticamente significativos.

E1 IMC de los participantes comprendidos entre 8-10 años alcanza como valor medio 18.89 y los de 11-13 años 19.63. Entre los dos grupos de edad hay una diferencia de .475 a favor de los de más edad.

Una vez reconvertidos los valores de las variables de satisfacción con las diferentes partes del cuerpo de una variable escala a una variable dicotómica, se ha aplicado la prueba $t$ de Student para muestras independientes para conocer si el hecho de estar satisfechos o no con cada una de las partes del cuerpo, peso y tono muscular puede inferir en algún factor.

El grado de satisfacción con parte inferior del cuerpo sí infieren los factores COM.PER $(t=-3.670, p<.001, \mathrm{DM}=.45)$, AUTOCONF $(t=-3.888, p<.001$, $\mathrm{DM}=.50)$ y C.ADEC $(t=-2.438, \quad p<.05, \quad \mathrm{DM}=.26)$, siendo los que están satisfechos los que puntúan más alto. Con los C.ERRO $(t=2.365, p<.05$, $\mathrm{DM}=.26)$, siendo los que están insatisfechos los que puntúan más alto.

Con la parte media del cuerpo los resultados indican que sí infieren los factores COM.PER $(t=-4.475, p<.001, \mathrm{DM}=.47)$, ATRC.FIS $(t=-2.728, p<.05$, $\mathrm{DM}=.32)$, AUTOCONF $(t=-3.805, p<.001, \mathrm{DM}=.43)$ y C.ADEC $(t=-3.162, p<.05$, $\mathrm{DM}=.29$ ), siendo los que están satisfechos los que puntúan más alto. 
Con la parte superior del cuerpo los resultados indican que sí infieren en los factores COM.PER $(t=-2.549, p .05, \mathrm{DM}=.34)$, FUER.FIS $(t=-2.127, p<.05$, $\mathrm{DM}=.30)$, AUTOCONF $(t=-3.673, p<.001, \mathrm{DM}=.50)$ y C.ADEC $(t=-3.180, p<.05$, $\mathrm{DM}=.36)$ siendo los que están satisfechos los que puntúan más alto.

Con el tono muscular los resultados indican que si infieren los factores COM.PER $(t=-3.135, p<.05, \mathrm{DM}=.34)$, FUER.FIS $(t=-5.311, p<.001, \mathrm{DM}=.56)$,

AUTOCONF $(t=-4.367, p<.001, \mathrm{DM}=.48)$ y C.ADEC $(t=-3.658, p<.001$, $\mathrm{DM}=.34)$, siendo los que están satisfechos los que puntúan más alto.

Con el peso resultados indican que sí infieren los factores COM.PER ( $t=-$ $5.577, p<.001, \mathrm{DM}=.53)$, ATRC.FIS $(t=-20.78, p<.05, \mathrm{DM}=.23)$, FUER.FIS $(t=-$ 2.972, $p<.05, \mathrm{DM}=.32)$, AUTOCONF $(t=-5.339, p<.001, \mathrm{DM}=.54)$ y C.ADEC $(t=-2.343, p<.05, \mathrm{DM}=.21)$ siendo los que están satisfechos los que puntúan más alto. Con los C.ERRO $(t=2.289, p<.05, \mathrm{DM}=.21)$, siendo los que están insatisfechos los que puntúan más alto.

Con su imagen general los resultados indican que si infieren en los factores COM.PER $(t=-2.782, p<.05, \mathrm{DM}=.43)$, ATRC.FIS $(t=-2.087, p<.05$, $\mathrm{DM}=.35)$, FUER.FIS $(t=-3.083, p<.05, \mathrm{DM}=.49)$ y AUTOCONF $(t=-2.608, p<.05$, $\mathrm{DM}=.42)$, siendo los que están satisfechos los que puntúan más alto.

Tabla 5

Prueba t de Student para conocer si el grado de satisfacción corporal infiere los factores

\begin{tabular}{|c|c|c|c|c|c|c|}
\hline & & \multicolumn{2}{|c|}{$\begin{array}{l}\text { Prueba de Levene de } \\
\text { calidad de varianzas }\end{array}$} & \multicolumn{3}{|c|}{ Prueba $t$ para la igualdad de medias } \\
\hline & & $\mathbf{F}$ & Sig. & $t_{(2,122)}$ & $p$ & DM \\
\hline \multirow{6}{*}{$\begin{array}{l}\text { Parte } \\
\text { Inferior }\end{array}$} & COM.PER & 1.037 & .311 & -3.670 & .000 & -.45 \\
\hline & ATRC.FIS & .000 & .984 & -1.307 & .194 & -.18 \\
\hline & FUER.FIS & .546 & .461 & -1.797 & .075 & -.24 \\
\hline & AUTOCONF & .783 & .378 & -3.888 & .000 & -.50 \\
\hline & C.ERRO & 2.361 & .127 & 2.365 & .020 & .26 \\
\hline & C.ADEC & .011 & .916 & -2.438 & .016 & -.26 \\
\hline \multirow{6}{*}{ Parte Media } & COM.PER & .324 & .571 & -4.475 & .000 & -.47 \\
\hline & ATRC.FIS & .007 & .933 & -2.728 & .007 & -.32 \\
\hline & FUER.FIS & .750 & .388 & -1.650 & .102 & -.19 \\
\hline & AUTOCONF & .611 & .436 & -3.805 & .000 & -.43 \\
\hline & C.ERRO & .448 & .505 & .331 & .741 & .03 \\
\hline & C.ADEC & .113 & .737 & -3.162 & .002 & -.29 \\
\hline \multirow{6}{*}{$\begin{array}{l}\text { Parte } \\
\text { Superior }\end{array}$} & COM.PER & .431 & .513 & -2.549 & .012 & -.34 \\
\hline & ATRC.FIS & .917 & .340 & -.167 & .868 & -.02 \\
\hline & FUER.FIS & .617 & .434 & -2.147 & .034 & -.30 \\
\hline & AUTOCONF & 1.091 & .298 & -3.673 & .000 & -.50 \\
\hline & C.ERRO & 1.974 & .163 & 1.535 & .127 & .18 \\
\hline & C.ADEC & .212 & .646 & -3.180 & .002 & -.36 \\
\hline \multirow{6}{*}{$\begin{array}{l}\text { Tono } \\
\text { Muscular }\end{array}$} & COM.PER & .003 & .959 & -3.135 & .002 & -.34 \\
\hline & ATRC.FIS & 2.000 & .160 & -1.709 & .090 & -.20 \\
\hline & FUER.FIS & .495 & .483 & -5.311 & .000 & -.56 \\
\hline & AUTOCONF & .160 & .690 & -4.367 & .000 & -.48 \\
\hline & C.ERRO & 1.635 & .203 & .249 & .804 & .02 \\
\hline & C.ADEC & .035 & .851 & -3.658 & .000 & -.34 \\
\hline \multirow{4}{*}{ Peso } & COM.PER & 1.695 & .195 & -5.577 & .000 & -.53 \\
\hline & ATRC.FIS & .612 & .436 & -2.078 & .040 & -.23 \\
\hline & FUER.FIS & 4.653 & .033 & -2.972 & .004 & -.32 \\
\hline & AUTOCONF & 1.815 & .180 & -5.339 & .000 & -.54 \\
\hline
\end{tabular}


Adriano Fernández Vallines y Carmen González González de Mesa

\begin{tabular}{|c|c|c|c|c|c|c|}
\hline & $\begin{array}{l}\text { C.ERRO } \\
\text { C.ADEC }\end{array}$ & $\begin{array}{l}.773 \\
.410 \\
\end{array}$ & $\begin{array}{l}.381 \\
.523\end{array}$ & $\begin{array}{r}2.289 \\
-2.343 \\
\end{array}$ & $\begin{array}{l}.024 \\
.021 \\
\end{array}$ & $\begin{array}{r}.21 \\
-.21 \\
\end{array}$ \\
\hline \multirow{6}{*}{$\begin{array}{l}\text { Satisfacción } \\
\text { general }\end{array}$} & COM.PER & 2.793 & .097 & -2.782 & .006 & -.43 \\
\hline & ATRC.FIS & .358 & .551 & -2.087 & .039 & -.35 \\
\hline & FUER.FIS & 2.440 & .121 & -3.083 & .003 & -.49 \\
\hline & AUTOCONF & .868 & .353 & -2.608 & .010 & -.42 \\
\hline & C.ERRO & .035 & .853 & .927 & .356 & .13 \\
\hline & C.ADEC & .763 & .384 & -1.780 & .077 & -.24 \\
\hline
\end{tabular}

Nota: Cuando las diferencias de medias son negativas quiere decir que puntúan más alto los que están satisfechos, y viceversa.

\section{Discusión y conclusiones}

En este estudio se han analizado diferentes factores como son el Autoconcepto, los Hábitos Alimentarios, la Imagen Corporal, el Índice de Masa Corporal y las horas semanales que dedican a realizar Actividad Física escolares que juegan al futbol. Realizan de forma regular entre 2-4 horas a la semana mínimo de actividad física moderada o vigorosa. Esto no asegura que cumplan con los 60 minutos diarios que se recomiendan, pero si que tiene relación con el mundo del deporte y la competición reglada (Escalante, 2011). Casi la mitad de encuestados reconocen que hacen más de 6 horas de actividad física a la semana siendo muy probable que lleguen al objetivo de 60 minutos al día. Esto puede darse por la implicación de las familias a que realice actividad física fuera de la escuela o porque sean activos y lo requieran.

A nivel nutricional el número de ingestas resultantes se considera óptima al ser más de los dos tercios de los sujetos encuestados los que afirma que realiza entre 4-5 ingestas diarias. Lo que cabria saber de ellas si cumplen los requisitos para ser saludables, y si compaginan el número de ingestas con uno de macronutrientes adecuados. La tendencia en la actualidad es comer dulces en el recreo de media mañana, y a media tarde alimentos con alto contenido en calorías y azúcares (Pérez-Llamas et al., 2004).

En relación a la baja participación de niñas en este estudio, fue totalmente casual/aleatorio, aunque la competición a estas edades sea mixta, en los clubs participantes no había más niñas. Esto puede deberse la escasa participación de mujeres en fútbol, los datos del Consejo Superior de Deportes, en el Anuario de Estadísticas Deportivas 2017 indica que mujeres federadas son solamente la cuarta parte de la totalidad.

A la cuestión de quien realiza la comida en casa, y en consecuencia quien es un pilar fundamental de la alimentación familiar, en la mitad de los 
hogares siguen cocinando las madres aunque un tercio dice que la comida la hacen ambos padres. Parece que el rol de ama de casa solo orientado a la mujer, va desapareciendo progresivamente (Fernández-Escárzaga y VázquezSoto, 2017), transformando la realidad del hogar e influyendo en algo tan relevante como es la nutrición de las nuevas generaciones (Criado, 2004).

Los factores de autoconcepto físico correlacionan entre sí presentando altos valores de significación (Barbosa y Urrea, 2018). Lo más relevante son los resultados de las correlaciones de los factores del autoconcepto con los conocimientos sobre hábitos alimentarios, donde un buen autoconcepto implica conocimientos adecuados, y tener menos conocimientos erróneos. Los conocimientos erróneos correlacionan de manera estadísticamente significativa en sentido negativo con la competencia percibida, el atractivo físico y la autoconfianza, lo cual revela que los que tienen conocimientos o creencias erróneas, puntúan menos en estos tres factores y viceversa (Rosa, 2015; Sánchez-Alcazar y Gómez-Marmos, 2014). Un estudio reciente sobre implementación de un programa de salud en adolescentes (Ceballos-Gurrola et al. 2020) concluyen que educación nutricional y ejercicio incide favorablemente sobre dimensiones del autoconcepto, lo cual reafirma la importancia de estos hallazgos, pues revalida la importancia de la educación nutricional en edades adolescentes.

Los resultados del IMC no correlacionan significativamente con ningún factor del autoconcepto ni con las horas de actividad fisica. Esto puede ser porque en los preadolescentes el nivel de desarrollo es muy grande, etapa donde se producen mayores cambios en peso, estatura y composición corporal grasa y muscular, además de la mineralización ósea (Burrows, Díaz, y Muzzo, 2004). Esto no indica que un IMC alto llegando a la obesidad en estas edades no sea preocupante, pero sí que se puede trabajar con un poco más de flexibilidad y utilizarlo como dato orientativo.

Se observa que las horas de práctica deportiva correlacionan con la competencia percibida y la autoconfianza, o sea, el aumento de horas de actividad física y práctica deportiva genera una sensación de competencia mayor y aumenta la confianza en uno mismo. Este resultado concuerda con los hallados por Ceballos-Gurrola et al. (2020) y Menéndez y González (2019).

En relación a la imagen corporal, cabe destacar que los valores de "cómo les gustaría ser" son más bajos que los de "cómo se ven". Esto indica que, ya sea por lo que entienden por cuerpo estandarizado, o por limitaciones físicas como estar más en forma, les gustaría estar más delgados, aun el grado de satisfacción con su cuerpo en la mayoría ronda el valor de 4 puntos sobre 7 , siendo estos valores muy positivos. El único valor que sobresale de forma negativa es la satisfacción con el peso (3.82 sobre 5) ya que suele ser un tema recurrente y muy gráfico, y una forma de control común de las familias y de los medios de comunicación, generando cierto grado de insatisfacción (Mancilla, Vázquez, Mancilla, Amaya y Álvarez, 2012). El hecho de estar satisfechos o no con cada una de las partes del cuerpo, el peso y tono 
muscular inferían en los factores de competencia percibida, atractivo físico, fuerza física y conocimientos adecuados, observando que los que estaban satisfechos con su cuerpo puntuaban más alto que los que no. Solo en el factor conocimientos erróneos puntuaban más alto los que estaban más insatisfechos. Un sentimiento de insatisfacción del cuerpo puede desencadenar en trastornos mentales que se deben prevenir desde la niñez, formando a jóvenes con conocimientos adecuados sobre alimentación y que practiquen deporte, para una mayor satisfacción con uno mismo (VaqueroCristóbal, Alacid, Muyor y López-Miñarro, 2013).

Como conclusiones se puede decir que la mayoría de estos escolares se acercan a la cantidad de horas semanales de actividad fisica que recomienda la OMS y mantienen un número óptimo de ingestas diarias; las personas con conocimientos sobre alimentación saludable manifiestan valores más altos en autoconcepto y percepción de la competencia; el mayor consumo de actividad física genera mejor percepción de la competencia y aumenta la confianza; y que aunque la imagen corporal es bastante buena, en general les gustaría estar más delgados.

Ante estos resultados es necesario insistir en la necesidad de implementar programas de intervención que hagan que la sociedad se conciencie en la importancia y necesidad de practicar actividad fisica de manera regular, adquirir los hábitos alimentarios pertinentes y conocimientos nutricionales adecuados, para prevenir las enfermedades, tanto físicas como psicológicas que avistan el futuro inmediato, producto de la ausencia de hábitos saludables, cuya carencia está muy extendida en la actualidad y que debe atajarse desde las edades tempranas.

\section{Referencias}

Barbosa, S. H. y Urrea, A. M. (2018). Influencia del deporte y la actividad física en el estado de salud físico y mental: una revisión bibliográfica. Katharsis, 25, 141-160.

Benarroch, A., Pérez, S. y Perales, J. (2011). Factores que influyen en las conductas alimentarias de los adolescentes: Aplicación y validación de un instrumento de diagnóstico. Electronic Journal of Research in Educational Psychology, 9(3), 1219-1232.

Benarroch, A. y Pérez, S. (2011). Hábitos e ingestas alimentarias de los adolescentes melillenses (España). Revistas Editorial Universidad de Granada, 41, 65-83.

Botella, L., Ribas, E. y Ruiz, J. B. (2009). Evaluación psicométrica de la imagen corporal: Validación de la versión española del Multidimensional Body Self Relations Questionnaire (MBSRQ). Revista Argentina de Clínica Psicológica, 18(3), 253-264.

Brown, T. A., Cash, T. F. y Mikulka, P. J. (1990). Attitudinal body-image 
assessment: Factor analysis of the Body-Self Relations Questionnaire. Journal of Personality Assessment, 55(1-2), 135-144.

Burrows, R., Díaz, N. y Muzzo, S. (2004). Variaciones del índice de masa corporal (IMC) de acuerdo al grado de desarrollo puberal alcanzado. Revista médica de Chile, 132(11), 1363-1368.

Busto, R., Amigo, I., Herrero, J. y Fernández, C. (2006). La relación entre la falta de sueño, el ocio sedentario y el sobrepeso infantil. Psicothema, 20(4), 516-520.

Carmona, C., Sánchez, P. y Bakieva, M. (2011). Actividades extraescolares y rendimiento académico: diferencias en autoconcepto y género. Revista de Investigación Educativa, 9(29), 447-465.

Ceballos-Gurrola, O., Lomas-Acosta, R., Enriquez-Martínez, M.A., Ramírez, E., Medina- Rodriguez, R.E. y Enriquez-Reyna, M.C. (2020). Impacto de un programa de salud sobre perfil metabólico y autoconcepto en adolescentes con obesidad. Retos, 38(2), 452-458.

Cash, T.F. (1990). Body image enhancement: A program for overcoming a negative body image. New York: Guilford.

Cenarruzabeitia, J. J. V., Hernández, J. A. M. y Martínez-González, M. Á. (2003). Beneficios de la actividad física y riesgos del sedentarismo. Medicina clinica, 121(17), 665-672.

Coballes, S. (2015). Relaciones entre actividad física, imagen corporal, autoconcepto e inteligencias múltiples de los adolescentes (Tesis Doctoral). Universidad de Oviedo. Oviedo. España.

Collins, M. E. (1991). Body figure perceptions and preferences among preadolescent children. International Journal of Eating Disorders, 1O(2), 199-208.

Consejo Superior de Deportes (2017). Anuario de estadisticas deportivas de 2017. Subdirección General de Estadística y Estudios. Ministerio de Educación Cultura y Deporte.

Criado, E. M. (2004). El valor de la buena madre. Oficio de ama de casa, alimentación y salud entre las mujeres de clases populares. Revista Española de Sociología, 4, 93-118.

Cuervo, C. (2016). Relación entre la imagen corporal, los hábitos alimentarios, el autoconcepto y la cantidad de práctica deportiva en jóvenes de 12 a 18 años (Tesis Doctoral). Universidad de Jaén, Jaén).

Cuervo, C., Cachón, J., Zagalaz, M.L. y González, C. (2018). Conocimientos e intereses sobre hábitos alimentarios saludables y práctica de actividad física. Un estudio con población adolescente. Aula Abierta, 47(2), 211-220. 
Escalante, Y. (2011). Actividad física, ejercicio físico y condición física en el ámbito de la salud pública. Revista Española de Salud Pública, 85(4), 325-328.

Fernández-Escárzaga, J. y Vázquez-Soto, M.A. (2017). La evolución de la familia y los estilos de educación. Revista Electrónica sobre Cuerpos Académicos y Grupos de Investigación 4(8), Recuperado en http: / / cagi.org.mx/index.php/CAGI/article/view/ 148

Fox, K. R. y Corbin, C. D. (1989). The Physical Self-Perception Profile: Development and preliminary validation. Journal of Sport and Exercise Psychology, 11, 408-430.

García, D. M., Lopez, I. G. y Eslava-Suanes, M. D. (2018). El fútbol como estrategia para el desarrollo de la inteligencia emocional del alumnado de educación primaria. Educação y Formação, 3(8), 17-36.

González-Pienda, J. A., Núñez, J. C., González-Pumariega, S. y García, M. S. (1997). Autoconcepto, autoestima y aprendizaje escolar. Psicothema, 9(2) 271-289.

Goñi, E. y Infante, G. (2015). Actividad físico-deportiva, autoconcepto físico y satisfacción con la vida. European Journal of Education and Psychology, 3(2), 199-208.

DOI: https://doi.org/10.30552/ejep.v3i2.52

Hanifi, R., Lambert, V. y Haskell, W. (2010). Recomendaciones mundiales sobre actividad física para la salud. Organización Mundial de la Salud.

http: / / apps.who.int/iris/bitstream/10665/44441/1/9789243599977_ spa.pdf

Hortigüela, D., Fernández-Río, J. y Pérez, Á. (2016). Efectos del planteamiento docente en la enseñanza del fútbol sobre el clima de aula: percepciones de alumnado y profesorado. Cuadernos de Psicología del Deporte, 16(1), 295-306.

Jiménez-Flores, P., Jiménez-Cruz, A. y Bacardí-Gascón, M. (2017). Insatisfacción con la imagen corporal en niños y adolescentes: revisión sistemática. Nutrición Hospitalaria, 34(2), 479-489.

Mancilla, A., Vázquez, R., Mancilla, J. M., Amaya, A. y Álvarez, G. (2012). Insatisfacción corporal en niños y preadolescentes: Una revisión sistemática. Revista mexicana de trastornos alimentarios, 3(1), 62-79.

Menéndez, D. y González, C. (2019). Relaciones entre la práctica de actividad física y deportiva, el autoconcepto, la imagen corporal y los hábitos alimentarios en estudiantes de primaria. E-Balonmano.com: Revista de Ciencias del Deporte, 15(1), 79-96.

Merino-Orozco, A., Arraiz-Pérez, A., Sabirón-Sierra, F., Usán-Supervía, P. 
y Jarie, L. (2017). La coordinación entre espacios formativos formales y deportivos: un estudio de caso etnográfico en el fútbol pre-benjamín. Ágora para la Educación Física y el Deporte, 19(2-3), 329-354.

DOI: https: / / doi.org/10.24197/aefd.2-3.2017.329-354

Moreno, J. A., Cervelló, E., Vera, J. A. y Ruiz, L. M. (2007). Physical selfconcept of Spanish schoolchildren: Diferences by gender, sport practicand levels of sport involvement. Journal of Education and Human Development, 1(2), 1-17.

http: / / www.scientificjournals.org/journals2007 / articles / 1 180.pdf

Muñoz, D. (2015). Insatisfacción corporal y práctica de actividad física y deportiva en adolescentes de Gipuzkoa (Tesis doctoral). Universidad del País Vasco, España.

Murillo-Godínez, G. y Pérez-Escamilla, L. M. (2008). Los mitos alimentarios y su efecto en la salud humana. Medicina Interna de México, 33(3):392-402.

Organización Mundial de la Salud. (2016). Informe de la Comisión para acabar con la obesidad Infantil. Ginebra, Suiza.

Organización Mundial de la Salud, (2017). Actividad física. Nota descriptiva. Ginebra, Suiza.

Pérez-Llamas, F., Garaulet, M., Herrero, F., Palma, J. T., Pérez de Heredia, F., Marín, R. y Zamora, S. (2004). Una aplicación informática multivalente para estudios del estado nutricional de grupos de población: Valoración de la ingesta alimentaria. Nutrición hospitalaria, 19(3), 160-166.

Pérez-Vadillo, S. (2013). Educación para una correcta alimentación en alumnos adolescentes. Diagnóstico de conductas alimentarias y propuesta de actividades de enseñanza-aprendizaje. Enseñanza de las ciencias: revista de investigación y experiencias didácticas, 31(3), 265273.

Rosa, A. (2015). Niveles de condición física y su relación con el perfil de autoconcepto en escolares de 8 a 11 años de la región de Murcia. EBalonmano.com: Revista de Ciencias del Deporte, 11 (3), 228-229.

Sánchez-Alcaraz, B. J. y Gómez-Mármol, A. (2014). Autoconcepto físico en una muestra de estudiantes de primaria y su relación con el género y la práctica deportiva extraescolar. E-Balonmano.com: Revista de Ciencias del Deporte, 10(2), 113-120.

Schilder, P. (2013). The image and appearance of the human body. Londres: Routledge.

Schmider, E., Ziegler, M., Danay, E., Beyer, L. y Bühner, M. (2010). Is it 
really robust? Reinvestigating the robustness of ANOVA against violations of the normal distribution assumption. Methodology, 6, 147151.

Sepúlveda, A. R., Gandarillas, A. y Carrobes, J. A. (2004). Prevalencia de trastornos del comportamiento alimentario en la población universitaria. $5^{\circ}$ Congreso Virtual de Psiquiatría. Recuperado de http: / / psiqu.com/2-6554

Soler, A. y Castañeda, C. (2017). Estilo de vida sedentario y consecuencias en la salud de los niños. Una revisión sobre el estado de la cuestión. Journal of Sport and Health Research, 9(2), 187-198.

Stunkard, A.J; Sorensen, T. y Schlusinger, F. (1983). Use of the Danish Adoption Register for the study of the obesity and thinness. In: Kety, L.P. Rowland; R. L, Sidman; S, y W; Mathysse (ed) Genetics of neurologic and physch disorders. New York: Raven Press, 115-120.

Vaquero, A., Macias, O. y Macazaga, A. (2014). La práctica corporal y la imagen corporal: reconstruyendo significados. Revista Electrónica Interuniversitaria de Formación del Profesorado, 17(1), 163-176. DOI: http://dx.doi.org/10.6018/reifop.17.1.181921

Vaquero-Cristóbal, R., Alacid, F., Muyor, J. M., y López-Miñarro, P. A. (2013). Imagen corporal: revisión bibliográfica. Nutrición hospitalaria, 28(1), 27-35. 